\title{
Ultrasound of tibialis anterior muscle and tendon: anatomy, technique of examination, normal and pathologic appearance
}

\author{
Ajay Varghese $\cdot$ Stefano Bianchi
}

Received: 1 November 2013/ Accepted: 12 November 2013/Published online: 19 December 2013

(C) Società Italiana di Ultrasonologia in Medicina e Biologia (SIUMB) 2013

\begin{abstract}
Lesions of the tibialis anterior muscle and tendon are not frequently reported in international literature although pathology is not rare. Pathology can be spontaneous, associated with arthropathy or more generalized conditions. Clinical assessment may not be sufficient for distinguishing conditions like tendinopathy, tears, bursitis, etc. Therefore, imaging studies are necessary to plan appropriate therapy. US has a number of advantages, including widespread availability, absence of contraindications and low cost. It can also be used for dynamic studies of the muscle during contraction and relaxation. This article reviews the anatomy of the tibialis anterior, normal variants, the technique used for standard US examination of this muscle and tendon, its normal appearance on US and the sonographic characteristics of the most common lesions that affect it including tips on US-guided injections used for treatment.
\end{abstract}

Keywords Tibialis anterior - Muscle - Tendon ·

Sonography

Riassunto Le lesioni del muscolo tibiale anteriore e del suo tendine non hanno un'ampia eco nella letteratura internazionale, anche se la patologia del tibiale anteriore non è rara. Questa può essere spontanea, associata ad artropatia o a condizioni più generali. La clinica spesso può non essere sufficiente per distinguere lesioni quali la tendinopatia,

\footnotetext{
A. Varghese

Dorset County Hospital NHS Foundation Trust, Dorchester, Dorset DT1 2JY, UK

A. Varghese $\cdot$ S. Bianchi $(\bowtie)$

CIM SA Cabinet Imagerie Médicale, route de Malagnou 40, 1208 Geneva, Switzerland

e-mail: cimgeneve@gmail.com
}

le rotture, le borsiti, ecc., quindi può essere necessario ricorrere alla diagnostica per immagini, per un appropriato trattamento terapeutico. L'ecografia ha numerosi vantaggi quali l'ampia diffusione, l'assenza di controindicazioni, il basso costo. Può essere utilizzata per studi dinamici con muscoli in contrazione e rilasciamento. Questo articolo rivaluta l'anatomia del tibiale anteriore, le varianti del normale, l'aspetto ecografico normale e le caratteristiche ecografiche delle lesioni più frequenti, comprende una trattazione dei trattamenti terapeutici ecoguidati.

\section{Introduction}

Lesions of the tibialis anterior muscle and tendon are not frequently reported in international literature although pathology like tibialis anterior tendinosis or rupture is not rare. Pathology can be spontaneous, related to trauma, associated with arthropathy or more generalized conditions like diabetes. In most cases, careful history taking and thorough physical examination are sufficient for making the diagnosis. Clinical assessment alone, however, may not be sufficient for distinguishing conditions like tendinopathy, tears, bursitis, etc. Therefore, at least in a proportion of patients, imaging studies are necessary to plan appropriate therapy that will allow prompt functional recovery. Radiography can be useful in conditions like myositis ossificans or to assess arthropathy. Magnetic resonance imaging, due to its excellent tissue contrast, allows simultaneous assessment of muscle, joint, and bone planes. It remains a second-line study due to its high cost and relatively low availability. It is also associated with various contraindications, the most important of which is the presence of a cardiac pacemaker. US has a number of advantages, including widespread 
availability, absence of contraindications and low cost. It can also be used for dynamic studies of the muscle during contraction and relaxation, and if doubts arise, scans can easily be obtained of the contralateral muscle for comparison purposes. These qualities make it an excellent tool for assessment and also follow-up of patients with tibialis anterior muscle and tendon lesions. This article reviews the anatomy of the tibialis anterior, normal variants, the technique used for standard US examination of this muscle and tendon, its normal appearance on US and the sonographic characteristics of the most common lesions that affect it including tips on US-guided injections used for treatment.

\section{Anatomy}

The tibialis anterior muscle has a prismatic belly that arises from the lateral condyle of the tibia, proximal one-third to two-thirds of the lateral surface of the tibial shaft, anterior surface of the interosseous membrane, deep surface of the fascia cruris and intermuscular septum [1]. The tibialis anterior tendon (TAT) begins at about the level of the junction between the lower and middle thirds of the tibia and courses towards the medial border of the foot, inserting vertically on the first metatarsal base and the medial cuneiform bone (Figs. 1, 2, 3). The tendon is the most medially located tendon in the ankle and foot. In its course, it is tightly and serially held against the front of the ankle and foot by the extensor retinaculum (ER) $[2,3]$. The ER covers the anterior aspect of the ankle and foot. It is a transverse aponeurotic band composed of both superior and inferior components.

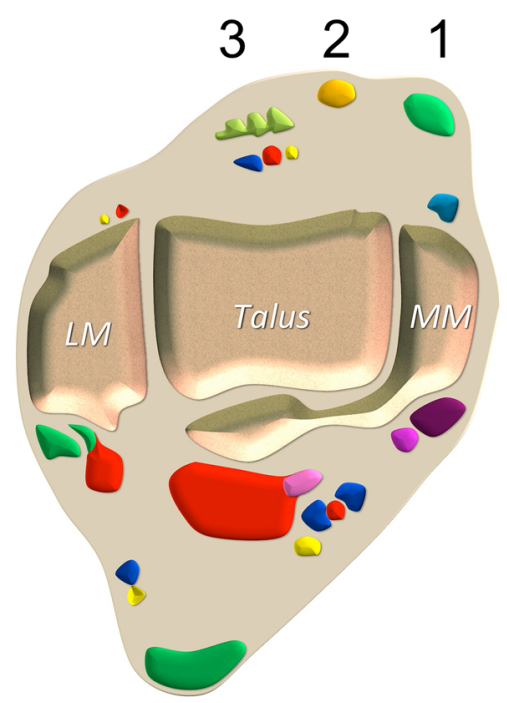

Fig. 1 Tibialis anterior tendon anatomy. Axial schematic drawing. 1 tibialis anterior tendon, 2 extensor hallucis longus tendon, 3 extensor digitorum longus tendon. $L M$ lateral malleolus, $M M$ medial malleolus

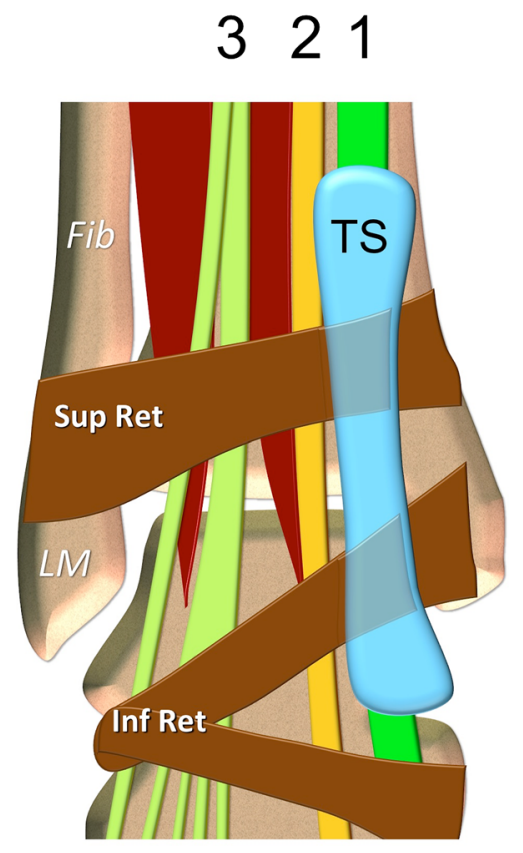

Fig. 2 Tibialis anterior tendon anatomy. Coronal schematic drawing. 1 tibialis anterior tendon, 2 extensor hallucis longus tendon, 3 extensor digitorum longus tendon. TS tendon sheath of the tibialis anterior tendon. LM lateral malleolus, Fib fibula. Sup Ret superior extensor retinaculum, Inf Ret inferior extensor tendons retinaculum

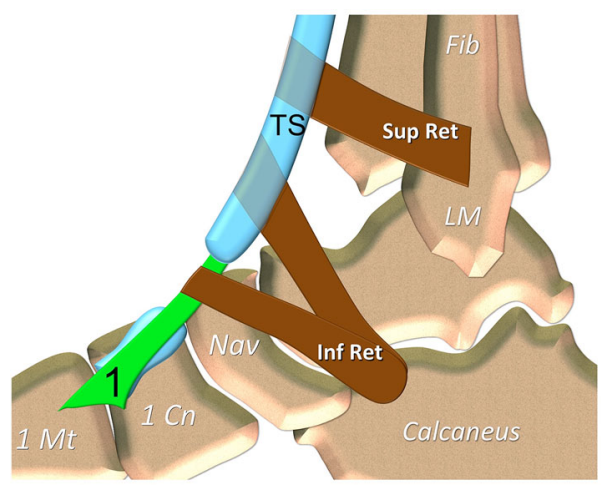

Fig. 3 Tibialis anterior tendon anatomy. Sagittal schematic drawing. 1 tibialis anterior tendon. TS tendon sheath of the tibialis anterior tendon. LM lateral malleolus, $1 \mathrm{Mt}$ first metatarsal, $1 \mathrm{Cn}$ medial cuneiform, Nav navicular, Fib fibula. Sup Ret superior extensor retinaculum, Inf Ret inferior extensor tendons retinaculum

The superior extensor retinaculum (SER) braces the tendons in the distal leg and the ankle. Its lateral attachment sites include the lateral crest of the lower fibula and the lateral surface of the lateral malleolus. Medially, it attaches to the anterior crest of the tibia and the medial malleolus. It is in continuity with the superior peroneal retinaculum laterally and with the flexor retinaculum medially. The extensor tendons pass beneath this 
retinaculum; however, in $25 \%$ of cases, there is a separate tunnel for the TAT formed by superficial and deep layers of the superior ER [2, 3].

The inferior extensor retinaculum (IER) is a complex Yor $\mathrm{X}$-shaped structure located in the anterior aspect of the foot and ankle comprising four components: the stem or frondiform ligament, the oblique superomedial limb, the oblique inferomedial limb, and the oblique superolateral limb. The stem is a sling ligament retaining the tendons of the extensor digitorum longus and peroneus tertius muscles against the talus and the calcaneus. Medially, it bifurcates into the oblique superomedial and inferomedial limbs. Laterally, it has three roots originating in the tarsal sinus and canal: lateral, intermediary, and medial [2,3]. The oblique superomedial limb continues from the stem and inserts in the anterior aspect of the medial malleolus. This limb passes over the extensor hallucis longus tendon, and medially, at the level of the TAT, it bifurcates into superior and inferior tunnels. The superior tunnel has a thick deep wall and a thin or even absent superficial wall. The inferior tunnel is well formed, with insertional fibers reaching the medial malleolus $[2,3]$. The synovial tendon sheath of the tibialis anterior extends from above the proximal arm of the inferior ER to the level of the talonavicular joint [4].

Different types of distal insertions have been described in the literature. Musial, in 1963, described four types [5]: type 1 , the second in frequency, with an insertion on the medial cuneiform and the base of the first metatarsal by two bands of the same width; type 2, the most common type, with a wide insertion on the medial cuneiform and a fine band of insertion to the base of the first metatarsal; type 3 with a wide insertion onto the medial cuneiform and some rare fibers to the base of the first metatarsal, and type 4 with a wide insertion to the base of the first metatarsal and a fine band inserting on the medial cuneiform. In 1990, Arthornthurasook identified three types [6]: type 1, the most common type, with two bands of equal dimension, respectively, on the medial cuneiform and base of first metatarsal; type 2, less common, with a broad band of insertion into the medial cuneiform and a small strip to the base of the first metatarsal and finally type 3, the rarer variety, with an insertion only on the medial cuneiform. In 2002, Brenner, meanwhile, described a broad band of insertion on the medial cuneiform and a small band of insertion on the base of the first metatarsal (45\%) two bands of equal width insertion ( $27 \%$ ), then a narrow band of insert on the medial cuneiform and a broad band distal insertion to the base of the first metatarsal (26\%). Other types of insertion-only on the medial cuneiform or only at the base of the first metatarsal-were present, but much more rare [7]. In most patients with distal tendinopathy, the band inserting into the medial cuneiform alone is affected.
Again in partial tear of the TAT the most commonly affected band is the one inserting into the medial cuneiform bone. All effort must be made by the sonologist to accurately assess both bands to avoid any misdiagnosis. The continuity and normal appearance of one band can lead the sonologist to miss a tear or severe tendinopathy affecting the other band, therefore, resulting in prolonged pain and disability. This is the reason why the sonologist should be aware of the different band insertions of the TAT with every attempt made to delineate them very well.

The main synovial bursa in association with the insertion of the TAT is located between the tendon and the cuneo-metatarsal joint and the medial cuneiform [8].

The tibialis anterior muscle is innervated by the deep peroneal nerve. Proximally, the blood supply of the TAT mainly arises from the anterior tibial artery. Distally, the tendon is supplied by the branches of the medial tarsal artery [9]. The tibialis anterior muscle is flexor, inverter (in addition to posterior tibial muscle) and adductor (in addition to the long extensor of hallux) of the foot. It also plays a role in suspension of the arch and controls supination of the rearfoot [10].

\section{US-technique}

The tibialis anterior muscle is best examined with a $10 \mathrm{MHz}$ or higher linear array transducer in both transverse and longitudinal planes from the origin just distal to the knee joint up to the tendon. Subjects are best positioned sitting with the hip in $90^{\circ}$ flexion and knee extended with the ankle unsupported. The limb should be exposed at least from mid thigh. Liberal application of coupling gel is advised over the skin and transducer head with the US beam aimed at $90^{\circ}$ to the muscle borders by visually ensuring the transducer is held perpendicular to the skin. Care should be taken to apply the minimum pressure possible so as not to distort the tissues with adjustments to the contrast, brightness and near and far gain. [11, 12].

Although the literature is poor, US is an excellent tool in assessing the TAT taking into account its very superficial and straight morphology. A linear high frequency probe (12-15 MHz at least) should be used. The optimal position would be with the foot flat on the examination couch with the patient seated or supine and knee flexed $45^{\circ}$. As a default setting, the field of view of the US image and the focal zone should be adjusted for the examination of small superficial parts with a high magnification scale and optimization of imaging parameters to increase spatial resolution. It can be evaluated in both long and short axis scanning planes from its myotendinous junction down to its bony insertion [13]. 


\section{US-anatomy}

The sonographic appearance of muscles is well known. In the transverse plane, perpendicular to the long axis of the muscle, the muscle has a speckled appearance because of reflections of perimysial connective tissue, which is moderately echogenic. In the longitudinal plane (along the long axis of the muscle) the fascicular architecture of the muscle becomes visible. Distinct reflections of the perimysial connective tissue in the tibialis anterior result in a pinnate structure on the US image. The boundaries of the muscle are clearly visible, as the epimysium surrounding the muscle is a highly reflective structure [14].

The normal tendon is hyperechoic with a fibrillated appearance with a diameter approximately twice that of the other extensor tendons (Fig. 4). In normal conditions, its synovial sheath cannot be distinguished even with a high frequency transducer. In normal states, retinacula are identified as thin bands that surround the tendons and insert into the periosteum. Owing to anisotropic properties, normal extensor retinacula appear hyperechoic over and hypoechoic at both sides of the underlying tendon. The lateral limbs of the retinacula may assume a curvilinear course deep to the restrained tendon and can be seen to insert onto the bone [13].

\section{Pathology}

Muscle disorders

\section{Herniation}

Most muscle hernias occur in the lower leg and affect the tibialis anterior muscle. Occupational and sporting activities, trauma, chronic compartment syndrome and weakness in the overlying fascia due to perforating vessels have been implicated as causes. Muscle protrudes through a defect in the fascia into the subcutaneous fat and presents clinically as a soft-tissue mass. Often there is a concern that the mass is a tumor. The sonographic features of muscle hernias are characteristic and exclude alternative clinical diagnoses such as tumors and muscle tears (Fig. 5). Advantages of sonography include the ability to examine the patient dynamically or erect and to show the nature of the lesion to the patient during the examination. Because surgical repair is not usually needed, visualizing the hernia on sonography helps to reassure the patient. A high frequency transducer should be used, and the gain and focus should be set to optimize the near field. The transducer should be applied lightly and a large amount of gel is used to avoid effacing the hernia. It may be necessary to get the patient to contract the muscle or to stand during the examination. Normal muscle is covered by thin echogenic fascia. Focal thinning and slight elevation of the fascia are subtle evidence of muscle hernias that can easily be overlooked. In most cases the margins of the defect are clearly defined and are easier to detect during dynamic examination [15-17].

\section{Atrophy}

In normal subjects, there can be asymmetry in muscle bulk depending on the effect of exercise, occupation and limb dominance $[11,18]$. US can be of value in the diagnosis of neuromuscular disorders by making it possible to reliably assess muscle thickness and objectify muscle atrophy (or hypertrophy). Besides muscle atrophy changes in muscle morphology can also be visualized with US (Fig. 6). Neuromuscular disorders can lead to increased muscle
Fig. 4 Tibialis anterior tendon US anatomy. a-d Transverse sonograms obtained from proximal to distal over the tibialis anterior tendon (arrows). Note how the tendon runs below the superior extensor retinaculum in (b) and in between a split of the retinaculum in (c). In (d) the arrowheads point to the inferior extensor retinaculum. Curved arrow extensor hallucis longus tendon, EHLm extensor hallucis longus muscle, $M C n$ medial cuneiform
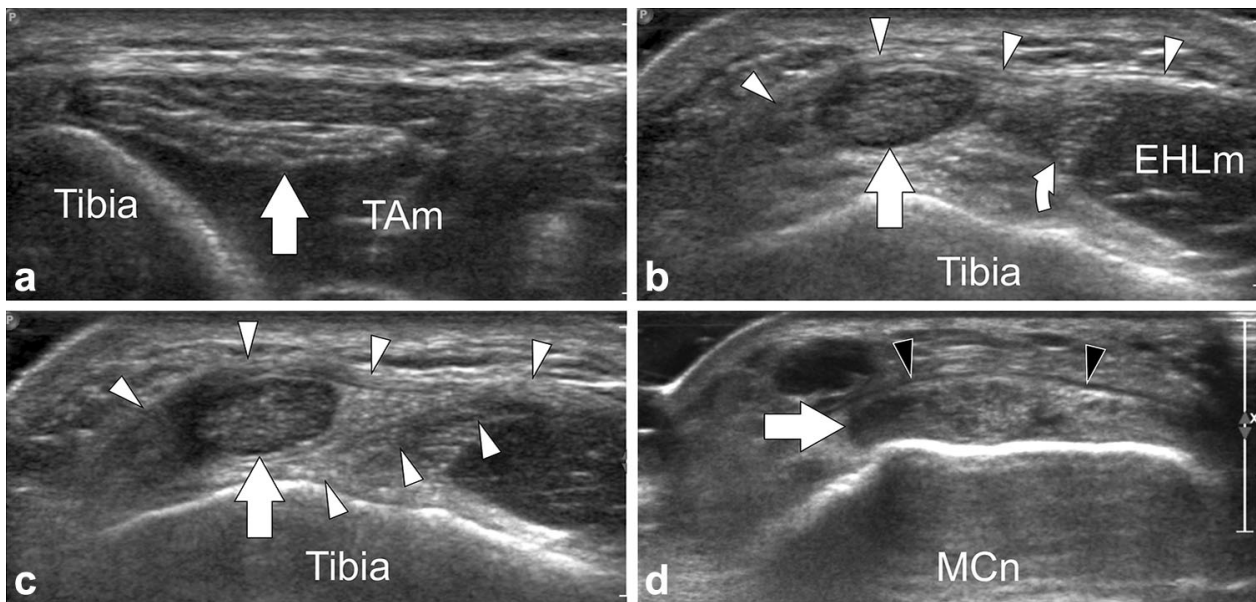
Fig. 5 Tibialis anterior muscle herniation. Axial sonograms obtained over the anterolateral aspect of the middle third of the leg, without (a) and with (b) local compression through the transducer, (b) longitudinal sonogram, (d) tangential radiograph. In (a) the anterior fascia (black arrow) of the tibialis anterior muscle (TAm) is torn and allows herniation of the muscle. In (b) local pressure reduces the herniation.

Longitudinal sonogram depicts the defect (black arrows) in the fascia. Radiograph shows focal bulging of the muscle
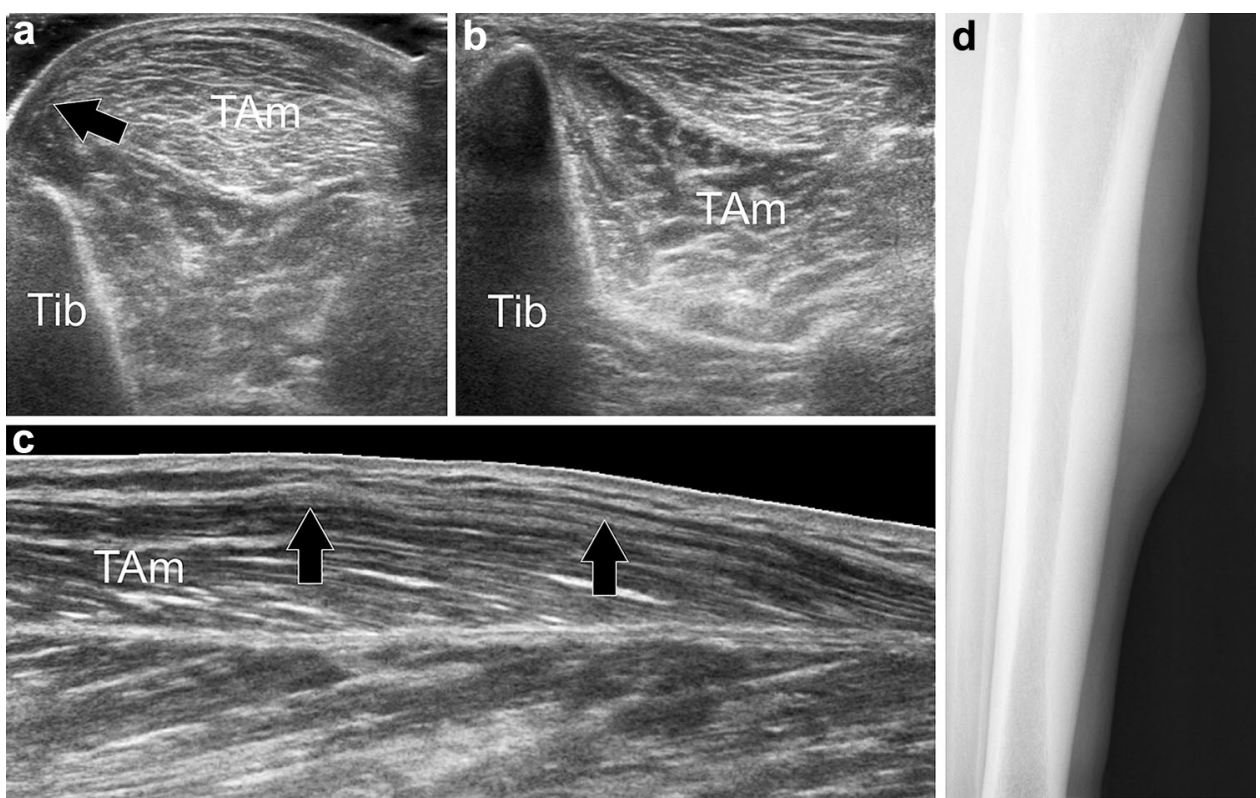
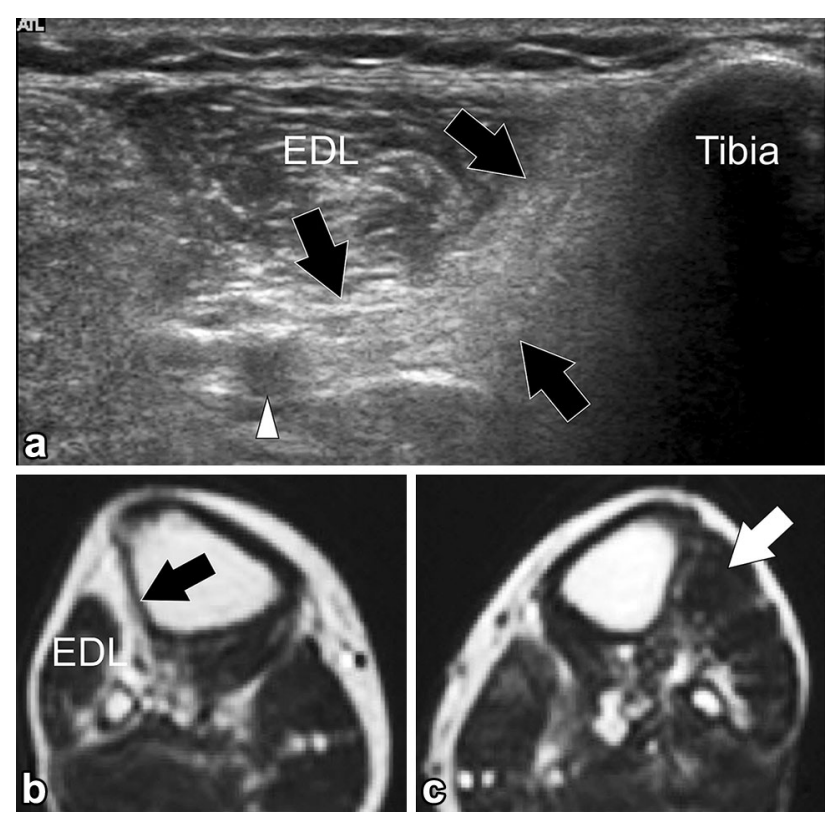

Fig. 6 Tibialis anterior muscle hypotrophy. a Axial sonogram obtained over the anterolateral aspect of the middle third of the leg. b Corresponding $\mathrm{T} 1$ weighted MR image, c normal contralateral MR image. In (a) the tibialis anterior muscle (black arrows) appears smaller than the adjacent extensor digitorum longus muscle (EDL). Note its hyperechoic appearance related to fat infiltration. MR image (b) correlates well with US. Note the normal tibialis anterior muscle (white arrow) in (c)

echo intensity, i.e., a muscle becomes whiter in appearance. It is thought that the replacement of muscle tissue with fat and fibrosis is the main cause of increased muscle echo intensity, as they increase the number of reflections within the muscle and therefore, the mean gray value of the muscle in the US image [14].
The causes of tibialis anterior atrophy would include common peroneal neuropathy due to direct trauma, knee dislocation, tight cast, prolonged squatting, fibular fracture, tumors, ganglia, etc. [19], in addition to more distal deep peroneal compromise or as part of a more global neuromuscular disorder.

\section{Calcification}

There are many causes of soft-tissue calcification which also affect the tibialis anterior muscle and tendon. Although rare, calcific myonecrosis needs special mention as it is inherently associated with diagnostic delays and difficulties. This is a condition that mainly affects the muscles of the lower leg including the tibialis anterior. Calcific myonecrosis is believed to be a late, uncommon sequela of blunt trauma, possibly associated with compartment syndrome and/or peroneal nerve injury. The affected muscle undergoes cystic degeneration or liquefaction, along with peripheral calcification and fibrosis. It presents as a tumor-like calcified mass years after an initial injury. Although the clinical, radiographic, and MRI features of calcific myonecrosis have been well described, sonographic description remains patchy [20, 21].

Finlay et al. [20] described a case of calcific myonecrosis which presented as a complex, tense, cystic mass in the anterior tibial muscle. The bulk of the mass had a background hypoechoic appearance, with uniform lowlevel echoes and mobile debris. A hyperechoic rim of uneven thickness was noted. Some internal irregular linear echoes were observed, many in a dependent location, some of which demonstrated posterior acoustic shadowing, suggesting calcifications. The large mass could be followed 
distally toward the anterior tibial tendon. The tendon was surrounded by thickened synovium, with hyperechoic foci of calcification identified within the tendon sheath in keeping with calcific tenosynovitis. Power Doppler analysis revealed hyperemia within the synovial lining [20].

The differential diagnosis of calcific myonecrosis is a calcified soft-tissue mass. This includes hematoma, synovial sarcoma, epithelioid sarcoma, soft-tissue osteosarcoma, and parosteal osteosarcoma. The differential diagnosis for calcification in soft tissue includes myositis ossificans, posttraumatic pseudoaneurysms, dermatomyositis/polymyositis, tumoral calcinosis, and diabetic myonecrosis $[21,22]$.

Tendon disorders

\section{Distal tendinopathy}

When compared to other tendinopathies in the ankle, for example involving the tibialis posterior or Achilles tendons, distal tendinopathy of the TAT is little reported in the literature and often little known about [23-25]. This is seen predominantly in overweight women of 50-70 years. The symptomatology includes burning on the medial side of the midfoot, often at night, with pain on palpation at the point of distal insertion. The pain is usually spontaneous, but in a few cases, has been described with previous trauma. Passive tension of the tendon causing pain is useful in the diagnosis (TAPS test or tibialis anterior passive stretch test) [23]. Ankle and foot are passively positioned in plantar flexion, eversion and abduction and pronation. The test is positive when the pain is reproduced or increased (this test can be compared to the Finkelstein test in de Quervain's tenosynovitis). The sensitivity of the TAPS is $90 \%$ with specificity of $95 \%$. Swelling next to the distal insertion is also frequently observed. The symptomatology is usually unilateral and there is no loss of strength, but active dorsiflexion of the foot is often limited. Mengiardi et al. [24] in a retrospective study of 28 patients noted an association between MRI abnormalities of the TA tendon and arthrosis of the first TMTJ, the medial naviculocuneiform joint or the talonavicular joint. The possible etiological relationship between insertional TA tendinosis and midfoot arthritis is unclear. Degenerative change in these joints is common, so it could be coincidental. Alternatively, osteophytes from these joints could abrade the distal section of the TAT as it passes over the arthritic joint. It is also possible that TAT might need to work harder in those patients with stiff arthritic midfoot joints. Macroscopically, the tendon is thickened, having lost its usual fibrillar architecture. A longitudinal split is often observed. Histologically, the tendon demonstrates hypervascularization and myxoid degeneration without
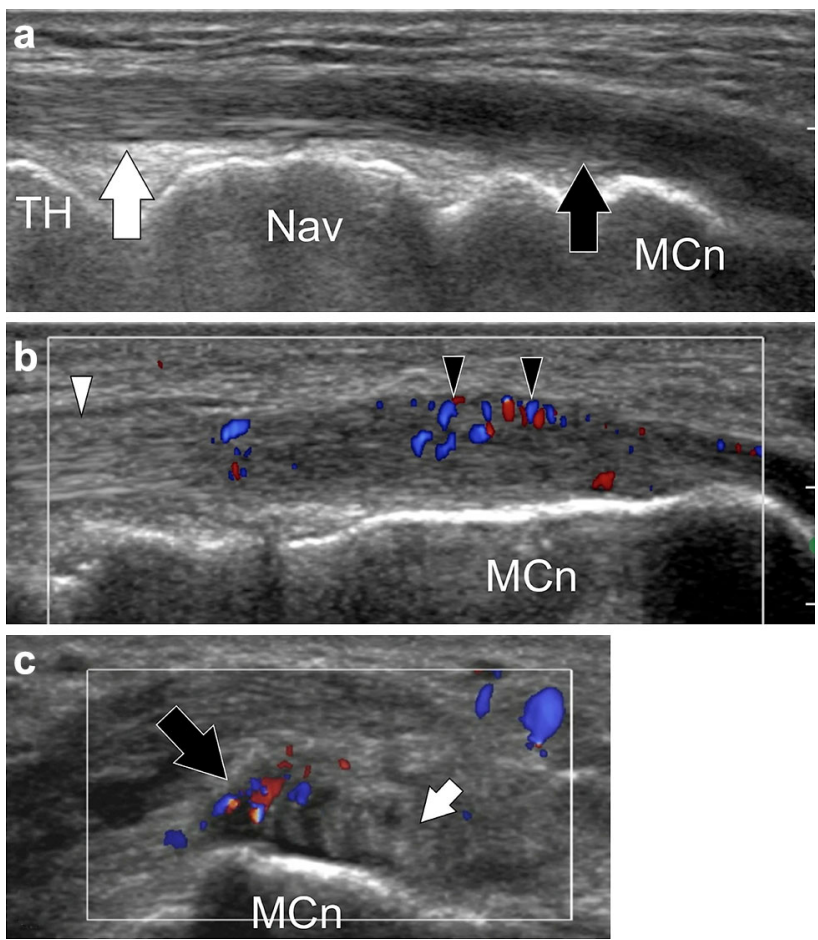

Fig. 7 Tibialis anterior distal tendinopathy. a, b Longitudinal grayscale and color Doppler sonograms obtained over the distal tibialis anterior tendon. (c) Transverse color Doppler sonogram. In (a) the middle tendon (white arrow) is normal, the distal tendon (black arrow) is swollen, irregular and hypoechoic. Note local hypervascular changes in (b). Transverse image shows the selective involvement of the tendon band inserting onto the medial cuneiform (MCn), the smaller tendon band inserting onto the first metatarsal is normal (small arrow)

inflammation or deposition of crystals [23-25]. Although the literature describing this is quite patchy, US is very useful in this scenario. Examination usually demonstrates irregular, hypoechoic swelling of the distal third of the tendon sometimes associated with hypervascular local changes at the insertion with longitudinal splits which may appear fluid filled. The inferior-medial part of the tendon or the deep proximal part is usually the part that is most affected (Fig. 7). This can be associated with effusion in the proximal synovial sheath. Distal bursitis also needs to be carefully evaluated for, which can be associated with the tendinopathy. The color Doppler can highlight hyperemia of the synovial sheath proximally or in region of the distal bursa [26].

\section{Tenosynovitis}

TAT tenosynovitis is a rare orthopedic condition that usually resolves with conservative treatment. It is difficult to clinically differentiate distal tendinopathy and tenosynovitis and can coexist. 
US is very often diagnostic and mirror the findings seen in tenosynovitis affecting other parts of the body. Sonographic features include:

- Edematous tendon thickening (compare with contralateral side) with hypoechogenicity, thickening of the synovial sheath, increased fluid within the tendon sheath seen in the upper synovial portion of the TAT.

- Peritendinous subcutaneous edema and/or hyperemia on Doppler imaging

In addition to the usual findings of tenosynovitis, unusual presentation with rice body formation has also been described in TAT nonspecific tenosynovitis [27]. Surgery may be required for chronic cases and endoscopy seems to be a valid therapeutic alternative. During debridement of the hypertrophic synovium, care must be taken to avoid damaging the ER to prevent potential postoperative bowstring phenomenon of the tendon [28].

\section{Tear}

TAT rupture is a relatively rare entity. There can be considerable delay in diagnosis due to misleading clinical findings. Approximately, 50 cases have been described in the orthopedic literature and around 10 cases in radiology literature. The rupture can be secondary to local trauma or spontaneous. Acute open or blunt trauma usually affects younger patients while spontaneous rupture usually affects middle-aged and old patients. Spontaneous tears are typically well tolerated by the patients who usually report slight local pain. In these cases, the rupture is mainly consequent to microtrauma acting on a weakened tendon. Either local or systemic diseases, namely corticosteroid injection, diabetes mellitus, hyperparathyroidism and gout as well as aging can decrease tendon strength [29-31].

At physical examination, the normally visible and palpable outline of the TAT, particularly in the forced dorsiflexion of the foot, cannot be appreciated. Instead, a firm soft-tissue mass, located on the anteromedial aspect of the distal leg is evident. This lump coincides with the retracted proximal portion of the lacerated tendon. There is usually no neurological deficit in these cases. The tear can go clinically unrecognized mainly because of preserved possibility of active dorsiflexion of the foot, which although significantly weakened, is usually still possible due to integrity of the extensor digitorum longus and extensor hallucis longus [32]. A review of the literature shows that often a definite diagnosis is missed at the first clinical examination. In patients with spontaneous tears this can be explained by the paucity of the symptoms and by the preserved ability to actively dorsiflex the foot. In acute cases other more significant lesions, such as fractures, could lead to unawareness of a tendon tear. Many cases undergo initial US evaluation because of a lump located at the anterior aspect of the ankle, assumed to be a "cyst".

According to the orthopedic literature, ATT tears are typically located within 5-30 $\mathrm{mm}$ from the point of insertion [29, 30, 33]. The etiology of ATT tears is still being debated. Although some investigators have reported homogeneous vascularity throughout the TAT [34], others consider an anterior area of hypovascularity in the tendon in the region of the retinacula to be a possible cause of ATT disorders. Petersen et al. [9] reported the presence of an avascular zone in the anterior half of the TAT that begins $5-16 \mathrm{~mm}$ from the insertion point and extends 45-67 $\mathrm{mm}$. Mengiardi et al. in their study on anterior tendon abnormalities on MRI scans do not support these theories. The signal intensity abnormalities in their study were most commonly found either in the posterior portion (39\%) of the tendon-close to the bone-or in a diffuse distribution (54\%); they were found in the anterior portion of the tendon in only $7 \%$ of cases. The significant association of bone morphologic features $(P=0.001$ for a bone spur on the navicular surface, $P<0.001$ for a ridged shape of the medial cuneiform bone, and $P=0.036$ for osteophytes at the medial tarsometatarsal joint) with TAT abnormalities indicates that a mechanical irritation of the tendon by bone structures may be pathogenetically relevant [24].

US reveals the palpable lump to correspond to the proximal end of the severed tendon, which appears irregular, hypoechoic and enlarged (Figs. 8, 9). The tendon fibers that normally have a regular fibrillar pattern appear disorganized and interrupted. Some cases can be associated with a synovial effusion in the tendon sheath which appears as a hypoechoic fluid collection surrounding the tendon. The extensor hallucis longus or extensor digitorum longus tendons are usually normal. An ankle joint effusion is usually not present.

\section{Bursal disorders}

\section{Bursitis}

A synovial bursa is a synovial-lined sac overlying the bony surfaces at areas of tendon friction [35-37]. Bursae are located where tendons move against each other or glide over a bony surface. They are classified according to their location: subcutaneous, subfascial, subtendinous, and submucosal [36]. Bursae can also be classified as communicating or noncommunicating. When a bursa is located adjacent to a joint, the synovial membrane of the bursae may communicate with the joint [36]. Most of the bursae are potential spaces and are not normally visualized on imaging. It has been well established that the TAT bursa is a subtendinous one deep to the extrasynovial portion of the tendon distally at the level of the medial cuneiform bone [37]. 

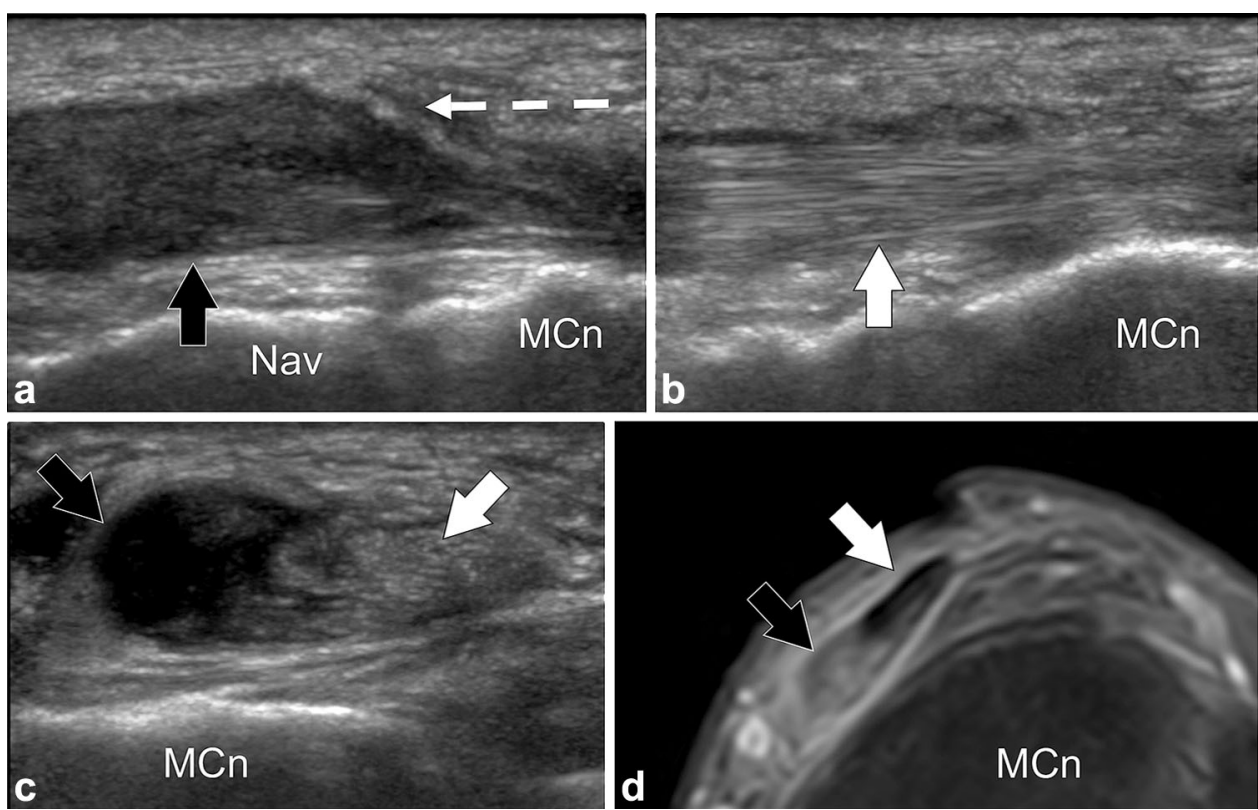

Fig. 8 Tibialis anterior partial tear. a, b Longitudinal gray-scale sonograms obtained over the two bands of distal tibialis anterior tendon. c Transverse sonogram. d MR STIR image corresponding to (c). In (a) the tendon band (black arrow) inserting onto the medial cuneiform $(\mathrm{MCn})$ is torn. The proximally tendon is swollen, hypoechoic and retracted (broken arrow) to the level of the tarsal navicular (Nav). In (b) the tendon band (white arrow) inserting onto the first metatarsal is normal. Transverse US and MR images (c, d) show selective tear of the tendon band inserting onto the medial cuneiform (black arrows), the tendon band inserting onto the first metatarsal is continuous (white arrows)

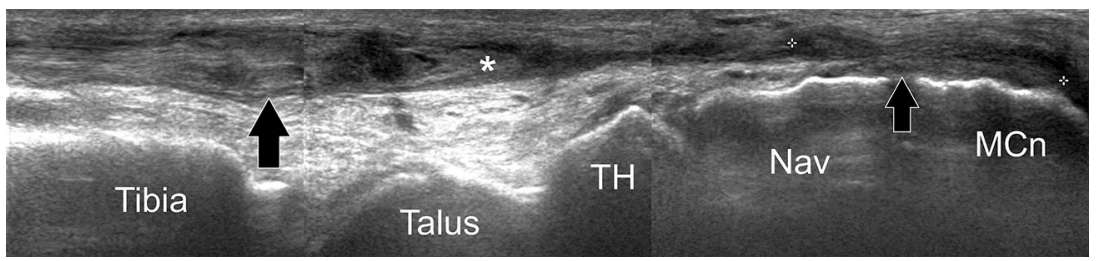

Fig. 9 Tibialis anterior complete tear. Longitudinal gray-scale sonogram of the tibialis anterior tendon shows the retracted irregular and swollen proximal tendon (large black arrow) and the distal

In pathological conditions such as excessive local friction, infection, arthritides or direct trauma, fluid and debris collect within the bursa or fluid extends into the bursa from the adjacent joint. The walls of the bursa thicken as the bursal inflammation becomes longstanding. The term bursitis refers to pathological enlargement of the bursa. If the bursa is abnormally distended, it is seen as a fluid-filled anechoic structure lined by a hyperechoic wall deep to the tendon (Fig. 10). Clinically, bursitis mimics several peripheral joint and muscle abnormalities. Therefore, it is important for the radiologist to identify bursal pathology and direct management geared toward bursitis [38].

\section{Injections}

Corticosteroids and local anesthetics can be injected into synovial cavities (joints, tendon sheaths, periarticular bursae) tendon stump (small black arrow). Note the tendon sheath filled by fluid and debris (asterisk). TH talar head, Nav navicular, MCn medial cuneiform

or peritendinous soft tissue. Previously injections were carried out under clinical guidance. Incorrect injection techniques can be painful for the patient and lead to accidental intra-tendinous injection of cortisone leading to possible tendinopathy or permanent injury. With the technological progress, new software and hardware, low cost and the dynamic nature of this method, US has in the recent years become a quick and safe procedure for guiding local corticosteroid injections (Fig. 11). The practical advantages of US guidance is the ability to confirm clinical diagnosis before local corticosteroid injection is administered, identify the most suitable approach on the basis of the anatomy and local pathology, monitor the progress of the needle in real-time and make sure that the drug is being injected into the appropriate location [39]. Use of US-guided steroid injections into the TAT sheath for severe tenosynovitis unresponsive to conservative management or for treatment of bursitis has been documented in the literature with more recent 
Fig. 10 Tibialis anterior bursitis. a-b Axial and longitudinal sonograms obtained over the distal tibialis anterior tendon. c Standard radiograph and (d) MR STIR image corresponding to (b). The distal tendon (white arrows) is normal. Bursitis is seen as a fluid collection (asterisks) located between the tendon and the joint plane. Radiograph shows a focal nonspecific softtissue swelling (arrowheads). MR shows the normal tendon partially surrounded by the fluid collection
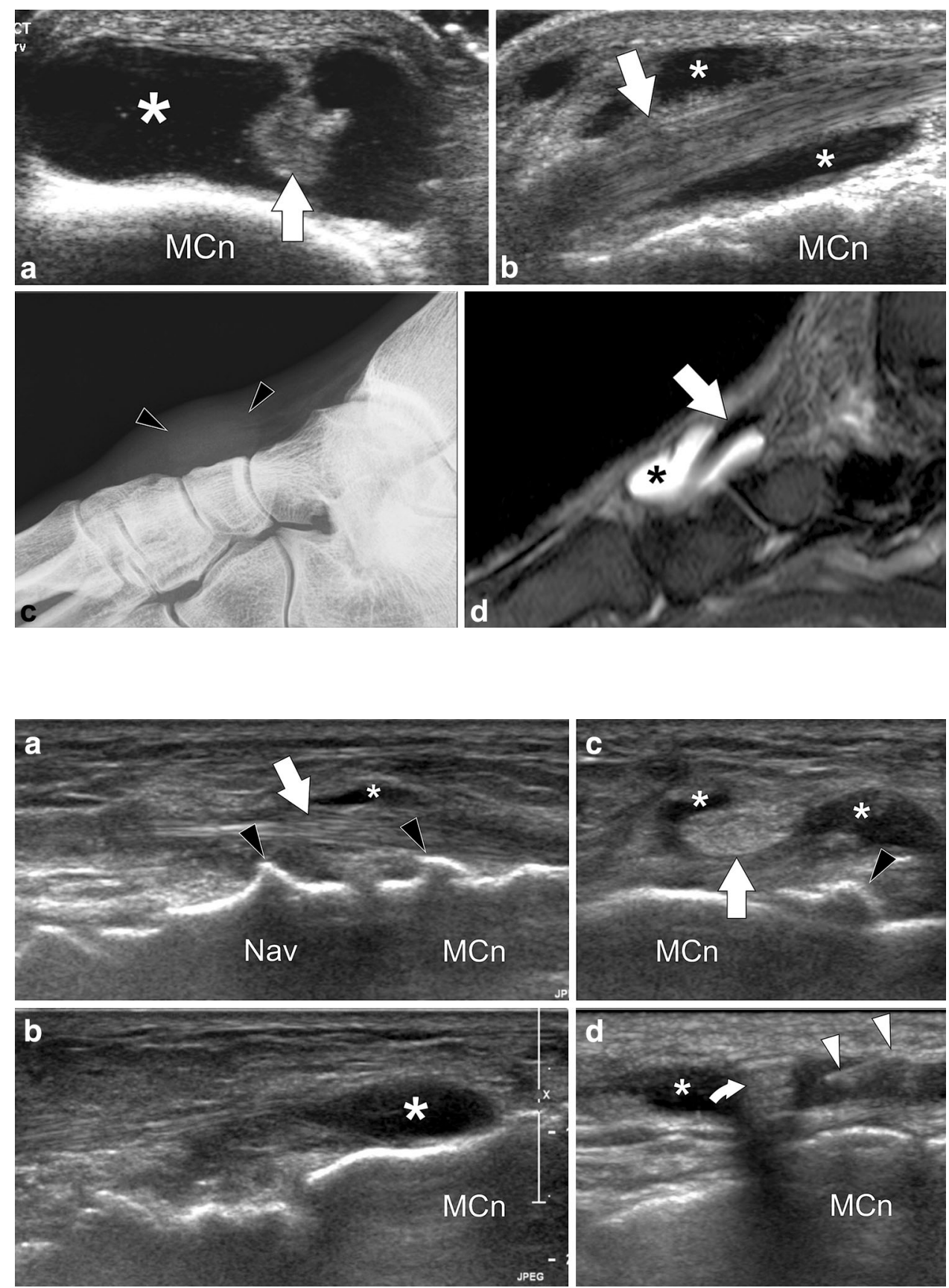

Fig. 11 Tibialis anterior distal bursitis. US-guided injection. a, b Longitudinal sonograms obtained over the distal tibialis anterior tendon and the adjacent bursitis. c Axial sonogram obtained before (c) and during (d) US-guided intrabursal injection. The tibialis anterior tendon (arrows) is normal. Note local bursitis (asterisks) probably due to local impingement on tarsal dorsal osteophytes (black arrowheads). In (d) US shows the needle (white arrowheads) correctly positioned inside the bursa. Note injected steroid with resultant posterior comet-tail artifact (curved arrow). Nav navicular, $M C n$ medial cuneiform

\section{Consent and technique}

studies by Laurell et al. [40] using this technique successfully in juvenile arthritis. They demonstrated excellent resolution of the tendon sheath effusion and synovial hypertrophy around the TAT 4 weeks after injection with follow-up US scans. Absolute contraindications include systemic or local ongoing infection putting the patient at high risk for sepsis, allergic reactions to the injectable or disinfectants used. Relative contraindications include diabetes, hypertension, treatment with anticoagulants and antiplatelet drugs, previous multiple corticosteroid injections, etc.
The procedure has to be explained to the patient with special mention about the potential side effects. If US examination precedes local corticosteroid injection, the usual US gel is substituted by a colorless liquid disinfectant to allow the skin to be in contact with the disinfectant during the whole procedure.

Most of the US-guided corticosteroid injections into the TAT sheath space or bursa performed in the authors' 
department are carried out with the patient reclining on the couch with his/her hip and knee in semi-flexion with the hip externally rotated. The physician is seated adjacent to the patient. When the most adequate position has been identified, the patient is asked to keep this position while the US probe and skin are disinfected. The authors do not use sterile US probe covers, but the probe is disinfected using the same disinfectant which is applied to the patient's skin.

With the probe in one hand and the syringe in the other, the skin is punctured after application of more disinfectant. The skin is always punctured at a distance from the probe to avoid contact between the needle and the probe. As soon as the needle has penetrated the subcutaneous tissues, its progress must be real-time monitored on the US image. When the US beam is perpendicular to the long axis, the needle is visible as a hyperechoic structure with posterior comet-tail artifact. The authors generally use a $3-5 \mathrm{~cm}$ long needle of 23-27 gage caliber. Thinner needles are more difficult to identify on the US image and furthermore, the thinner the needle the more difficult it is to perform the injection. Delayed-release corticosteroids are injected because of their long-lasting effect. A local anesthetic (lidocaine $1 \%$ ) can be used for an analgesic effect. The authors inject a dose of steroids mixed with $0.5-1 \mathrm{cc}$ of $1 \%$ lidocaine solution.

\section{Complications}

The most severe complication is the onset of infection. This is a very rare complication but may require treatment with antibiotics. A temporary vasovagal reaction is a wellrecognized complication. A painful reaction may complicate local corticosteroid injection related to microcrystalline synovitis in cases where delayed-action corticosteroid has been used. The pain will disappear spontaneously or upon administration of analgesic medication. Flushing of the skin may occur 2-3 days after corticosteroid injection, sometimes associated with a warm sensation in the cheeks. On rare occasions there may be skin atrophy or depigmentation at the injection site if the corticosteroid injection was performed in a too superficial, subcutaneous site [39].

\section{Conclusion}

A good understanding of the normal anatomy and its variants becomes necessary when dealing with pathology related to tibialis anterior muscle and tendon. Clinical assessment is indispensable and provides clues as to the most appropriate diagnostic studies. When US is performed by adequately trained personnel with high-quality equipment, it offers excellent visualization of the tibialis anterior muscle and tendon. In vast majority of cases US is the only examination performed. Delayed and missed diagnosis are recognized in pathology like tibialis anterior rupture which should prompt the clinicians to make use of this relatively easily available and less costly resource more when dealing with tibialis anterior pathology. There are well-recognized indications for corticosteroid and anesthetic injections to treat various TAT and bursal conditions with proven improvement in efficacy if the procedure is done under real-time US guidance. In short US offers a very efficient imaging modality to complement clinical examination in the diagnosis and management of various pathologies involving the tibialis anterior muscle and tendon.

Conflict of interest Ajay Varghese and Stefano Bianchi declare that they have no conflict of interest.

Human and animal studies The study described in this article did not include any procedures involving humans or animals.

\section{References}

1. Borley NR, Healy JC, Collins P, Johnson D, Crossman AR, Mahadevan V et al (2008) Leg. In: Standring S (ed) Grays anatomy, 40th edn. Churchill Livingstone Elsevier, New York, p 1417

2. Saraffian SK (1993) Retaining systems and compartments. In: Saraffian SK, Kelikian AS (eds) Anatomy of the foot and ankle: descriptive, topographic, functional. Lippincott, Philadelphia, pp 113-118

3. Lee MH, Chung CB, Cho JH, Mohana-Borges AV, Pretterklieber ML, Trudell DJ, et al (2006) Tibialis anterior tendon and extensor retinaculum: imaging in cadavers and patients with tendon tear. AJR 187(2):W161-8

4. Saraffian SK, Kelikian AS (2011) Tendon sheaths and bursae. In: Saraffian SK, Kelikian AS (eds) Anatomy of the foot and ankle: descriptive, topographic, functional. Lippincott, Philadelphia, p 292

5. Musial WW (1963) Variations of the terminal insertions of the anterior and posterior tibial muscles in man. Folia Morphol 22:237-247

6. Arthornthurasook A, Gaew Im K (1990) Anterior tibial tendon insertion: an anatomical study. J Med Assoc Thai 73:692-696

7. Brenner E (2002) Insertion of the tendon of the tibialis anterior muscle in feet with and without hallux valgus. Clin Anat $15: 217-223$

8. Bianchi S, Martinoli C, Gaignot C, De Gautard R, Meyer JM (2005) Ultrasound of the ankle: anatomy of the tendons, bursae and ligaments. Semin Musculoskelet Radiol 9:243-259

9. Petersen W, Stein V, Tillmann B (1999) Blood supply of the tibialis anterior tendon. Arch Orthop Trauma Surg 119:371-375

10. Piat C, Wybier M (2010) Le tendon tibial antérieur. Entretiens de Bichat 8-12

11. McCreesh K, Egan S (2011) Ultrasound measurement of the size of the anterior tibial muscle group: the effect of exercise and leg dominance. Sports Med Arthrosc Rehabil Ther Technol 3:18

12. Martinson H, Stokes MJ (1991) Measurement of anterior tibial muscle size using real-time ultrasound imaging. Eur J App Physiol 63:250-254

13. Bianchi S, Martinoli C (2007) Ankle. In: Ultrasound of the musculoskeletal system. Springer pp 773-834 
14. Pillen S (2010) Skeletal muscle ultrasound. Eur J Transl Myol $1: 145-155$

15. Bianchi S, Abdelwahab IF, Mazzola CG, Ricci G, Damiani S (1995) Sonographic examination of muscle herniation. J Ultrasound Med 14:357-360

16. Beggs I (2003) Sonography of muscle hernias. AJR 2:395-399

17. Bates DG (2001) Dynamic ultrasound findings of bilateral anterior tibialis muscle herniation in a pediatric patient. Pediatr Radiol 31:753-755

18. Bianchi S, Martinoli C (2007) Muscle and tendon. In: Ultrasound of the musculoskeletal system. Springer pp 45-97

19. Kim SJ, Hong SH, Jun WS et al (2011) MR imaging mapping of skeletal muscle denervation in entrapment and compressive neuropathies. Radiographics 31:319-332

20. Finlay K, Friedman L, Ainsworth K (2007) Calcific myonecrosis and tenosynovitis: sonographic findings with correlative imaging. J Clin Ultrasound 35:48-51

21. O’Dwyer HM, Al-Nakshabandi NA, Al-Muzahmi K, Ryan A, O'Connell JX, Munk PL (2006) Calcific myonecrosis: keys to recognition and management. AJR 187:W67-W76

22. Banks KP, Bui-Mansfield LT, Chew FS, Collinson F (2005) A compartmental approach to the radiographic evaluation of soft tissue calcifications. Semin Roengenol 40(4):391-407

23. Beischer AD, Beamond BM, Jowett AJ, O'Sullivan R (2009) Distal tendinosis of the tibialis anterior tendon. Foot Ankle Int 30:1053-1059

24. Mengiardi B, Pfirrmann CW, Vienne P et al (2005) Anterior tibial tendon abnormalities: MR imaging findings. Radiology 235: 977-984

25. Grundy JR, O'Sullivan RM, Beischer AD et al (2010) Operative management of distal tibialis anterior tendinopathy. Foot Ankle Int 31:212-219

26. Jacob D, Bianchi S (2011) Le tendon tibial antérieur. Societe D'Imagerie Musculo-squelettique. Opus XXXVIII Le pied

27. Bulut M, Yilmaz E, Karakurt L, Özercan MR (2013) Rice body formation characterized by the chronic non-specific tenosynovitis in the tibialis anterior tendon. Acta Orthop Traumatol Turc 47:142-145
28. Maquirriain J, Sammartino M, Ghisi JP, Mazzuco J (2003) Tibialis anterior tenosynovitis: avoiding extensor retinaculum damage during endoscopic debridement. Arthroscopy 19:E9

29. Bianchi S, Zwass A, Abdelwahab IF, Zoccola C (1994) Evaluation of tibialis anterior tendon rupture by ultrasonography. J Clin Ultrasound 22:564-566

30. Gallo RA, Kolman BH, Daffner RH, Sciulli RL, Roberts CC, DeMeo PJ (2004) MRI of tibialis anterior tendon rupture. Skeletal Radiol 33:102-106

31. Khoury NJ, el-Khoury GY, Saltzman CL, Brandser EA (1996) Rupture of the anterior tibial tendon: diagnosis by MR imaging. AJR 167:351-354

32. Wulker N, Hurschler C, Emmerich J (2003) In vitro simulation of stance phase gait. II: simulated anterior tibial tendon dysfunction and potential compensation. Foot Ankle Int 24:623-629

33. Dooley BJ, Kudelka P, Menelaus MB (1980) Subcutaneous rupture of the tendon of tibialis anterior. J Bone Jt Surg Br $62-\mathrm{B}: 471-2$

34. Geppert MJ, Sobel M, Hannafin JA (1993) Microvasculature of the tibialis anterior tendon. Foot Ankle 14:261-264

35. Resnick D (1995) Diagnosis of bone and joint disorders. 3rd edn. W. B. Saunders, Philadelphia, pp 667-8

36. Farooki S, Ashman C, Lee J, Guttikonda S, Yu J (2002) Common bursae around the body: a review of normal anatomy and magnetic resonance imaging findings. Radiologist 9:209-211

37. Pocket Atlas of Human Anatomy: Founded by Heinz Feneis Basic Sciences (Thieme) Dauber 5th Rev edn. pp 132-133

38. Hirji Z, Hunjun JS, Choudur HN (2011) Imaging of the Bursae. J Clin Imaging Sci 1:22

39. Molini L, Mariacher S, Bianchi S (2012) US guided corticosteroid injection into the subacromial-subdeltoid bursa: technique and approach. J Ultrasound 15:61-68

40. Laurell L, Court-Payen M, Nielsen S, Zak M, Boesen M, Fasth A (2011) Ultrasonography and color Doppler in juvenile idiopathic arthritis: diagnosis and follow-up of ultrasound-guided steroid injection in the ankle region. A descriptive interventional study. Pediatr Rheumatol Online J 9:4 